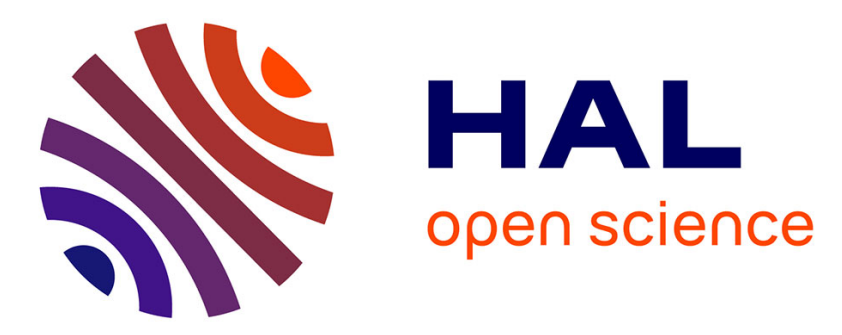

\title{
Perturbation series for shear stress in flexure of Saint-Venant Cylinders with Bredt-like sections
}

\author{
Francesco Dell'Isola, Giuseppe C. Ruta
}

\section{To cite this version:}

Francesco Dell'Isola, Giuseppe C. Ruta. Perturbation series for shear stress in flexure of Saint-Venant

Cylinders with Bredt-like sections. Mechanics Research Communications, 1996, pp.8. hal-00502011

\section{HAL Id: hal-00502011 \\ https://hal.science/hal-00502011}

Submitted on 13 Jul 2010

HAL is a multi-disciplinary open access archive for the deposit and dissemination of scientific research documents, whether they are published or not. The documents may come from teaching and research institutions in France or abroad, or from public or private research centers.
L'archive ouverte pluridisciplinaire HAL, est destinée au dépôt et à la diffusion de documents scientifiques de niveau recherche, publiés ou non, émanant des établissements d'enseignement et de recherche français ou étrangers, des laboratoires publics ou privés. 


\section{PERTURBATION SERIES FOR SHEAR STRESS IN FLEXURE OF SAINT-VENANT CYLINDERS WITH BREDT-LIKE SECTIONS}

Francesco dell'ISOLA $\ddagger$ and Giuseppe Claudio RUTA $\dagger$

$\ddagger$ Dip. di Ingegneria Strutturale e Geotecnica, †Dottorato di Ricerca in Meccanica Teorica e Applicata

Università di Roma "La Sapienza", Facoltà di Ingegneria, via Eudossiana 18, I-00184 Roma

(Received 20 February 1996; accepted for print 24 July 1996)

Introduction

We study flexure of a Saint-Venant cylinder whose sections are Bredt-like with constant thickness, as defined in [1], [2], [3]. We propose a perturbation series for the shear stress field in terms of a thickness parameter. In torsion, there is a unique stress flow function (named after Prandtl) for any regular section ([3], [4], [6], [7]). On the contrary, in flexure of cylinders with bi-connected sections such a flow function cannot be uniquely determined. To avoid a gauge choice, we use different perturbation methods for mono- and bi-connected sections. In the last case we introduce a perturbation series directly for shear stress and Helmholtz differential operators. For both classes of sections the resulting hierarchy of problems for the series terms can be solved. The perturbation series in the present form does not always converge ([10]); however, it allows for a rational deduction of many of the engineering formulæ found in technical textbooks (e.g. Jourawski formula, [6], [7], [12], [13]). Moreover, the method we propose seems to frame many of the results on thin walled bars quoted in [12], without the need for introducing internal constraints of Kirchhoff type. This is actually an interesting result, because we prove that the introduction of such constraints is not necessary a priori: in fact, our procedure of asymptotic development shows that the solution of Saint-Venant flexure problem always verifies constraints of Kirchhoff type when the section is thin.

The method presented here supplies: i) an efficient procedure for comparing the order of magnitude, in terms of the thickness parameter, of the stress field components and resultants; ii) a probably useful method for finding new results for thick sections considering higher order terms in the perturbation parameter: we show these results for some sections of technological interest; iii) as examples we consider rectangular and annular sections, in order to give an estimate of the relative magnitude of the maximum values attained by the stress components. For instance, an interesting caveat can be obtained: when the radius of curvature is five times the thickness, the second order maximum tangential stress component amounts to $15 \%$ of that given by Jourawski formula. As a by-product we are able, in the case of mono-connected sections, to estimate the boundary layer effect of Kelvin type ([11]) in flexure.

We remark that: a) our series supply analytic expressions for shear stress which can be, once regularized, of some use in technological applications, especially in optimization problems; b) our results open interesting mathematical problems like those solved by Wheeler and Horgan for the lowest term of shear stress in the case of mono-connected sections ([1], [2]). All calculation will be performed in absolute notation, for sake of simplicity; the most important results will also be given in components along an orthonormal basis, to be of more immediate grasp. 
Geometry of Bredt-like sections

We adopt a section, called Bredt-like in [3], obtained by thickening a plane curve $\mathcal{L}$ (middle line) along its Frenet normal with constant thickness. The middle line is given in terms of its arc length $s$ :

$$
\mathcal{L}:=\left\{q \in \mathcal{P} \mid q-m=\mathbf{r}_{0}(s), s \in[0, l]\right\}
$$

$m$ is the centroid of $\mathcal{L}$ and $\mathcal{P}$ is the plane including the section. The Frenet basis for the middle line is:

$$
\mathrm{I}(s):=\frac{\partial \mathbf{r}_{0}}{\partial s}=\dot{\mathbf{r}}_{0}(s), \quad \mathbf{m}(s):=-* \frac{\partial \mathbf{r}_{0}}{\partial s}=-* \dot{\mathbf{r}}_{0}(s)
$$

* is Hodge operator in $\mathcal{P}\left(\frac{\pi}{2}\right.$ rotation in the positive orientation of $\mathcal{P}$ ). The $\epsilon$-Bredt-like section is given by

$$
\mathcal{D}_{\epsilon}:=\left\{y \in \mathcal{P} \mid y-m=\mathbf{r}(s, z)=\mathbf{r}_{0}(s)+z \epsilon \delta * \dot{\mathbf{r}}_{0}(s), s \in[0, l], z \in[-1,1]\right\}
$$

In (3) $z$ is the coordinate along $* \dot{\mathbf{r}}_{0}, \delta$ is half the thickness of the section and $\epsilon$ is a thickness parameter playing the role of the perturbation parameter. We regard $s, z$ as rescaled coordinates over $\mathcal{D}_{\epsilon}([8])$.

The field problem for the shear stress $t$ in flexure is written in the form $([5],[6])$

$$
\begin{aligned}
\operatorname{div} \mathbf{t} & =Y \mathbf{k} \cdot[*(y-b)] \quad \text { in } \mathcal{D}^{o} \\
\operatorname{curl} \mathbf{t} & =2 G \nu \mathbf{k} \cdot(y-o) \quad \text { in } \mathcal{D}^{\circ} \\
\mathbf{t} \cdot \mathbf{n} & =0 \quad \text { on } \partial \mathcal{D} \\
\oint_{\mathcal{L}=\partial \mathcal{R}}(\mathbf{t} \cdot \mathbf{l}) d s & =2 G \nu \mathbf{k} \cdot A_{\mathcal{R}}\left(b_{\mathcal{R}}-o\right) .
\end{aligned}
$$

In (4)-(7) $Y, G, \nu$ are Young's and shear moduli and Poisson's ratio, respectively; $\mathbf{k}$ describes the linear variation of the curvature of the axis of the cylinder $([6]) ; \mathcal{R}$ is the inner Jordan region of $\mathcal{L}$ and its area is $A_{\mathcal{R}} ; b$ and $b_{\mathcal{R}}$ are the centroids of $\mathcal{D}$ and $\mathcal{R}$, respectively; $o$ is any point in $\mathcal{P}$. Let $\mathbf{t}_{o}, \mathbf{t}_{o^{\prime}}$ be the solutions of (4)-(7) associated to $o$ and $o^{\prime}$, respectively. The field $\mathbf{t}_{o}-\mathbf{t}_{o^{\prime}}$ is the solution of a torsion problem, already studied in [3]. Because of (3) we obtain

$$
b-o=m-o-\frac{\epsilon^{2} \delta^{2}}{3 l}\left|\dot{\mathbf{r}}_{0}\right|_{0}^{l}=: m-o-\frac{\epsilon^{2} \delta^{2}}{3 l} \mathbf{v} .
$$

Following Timoshenko [11], we will choose $b=0$. The resultant of the shear stress field solution of (4)-(7) is

$$
\mathbf{q}: \stackrel{(1)}{=} \int_{\mathcal{D}} \mathbf{t} d \stackrel{(2)}{=} Y * \mathbf{J}_{b} \mathbf{k}, \quad \mathbf{J}_{b}: \stackrel{(3)}{=} \int_{\mathcal{D}}\left[\left(* \mathbf{r}_{b}\right) \otimes\left(* \mathbf{r}_{b}\right)\right] d \mathcal{D}
$$

where $\mathbf{r}_{b}$ is the position vector field with respect to the centroid $b$. Eq. (9) 2 derives from global balance equations of force and torque on the cylinder. Let us consider an orthonormal basis $\left(\mathbf{e}_{1}, \mathbf{e}_{2}\right)$ along the directions of the principal axes of inertia of the section; let us also consider a rectangular Cartesian coordinate system with origin $b$ and $x$ and $y$ axes along $\mathbf{e}_{1}, \mathbf{e}_{2}$. Then the tensor of inertia defined by Eq. $(9)_{3}$ is represented by the matrix

$$
\mathbf{J}_{b}=\left(\begin{array}{cc}
I_{x} & 0 \\
0 & I_{y}
\end{array}\right),
$$

where $I_{x}, I_{y}$ are the moments of inertia of the section with respect to the $x$ and $y$ axes, respectively. The parameter $\mathbf{k}$ is given in terms of the components $q_{1}, q_{2}$ of the resultant shear force by (for instance see [6])

$$
\mathbf{k}=-\frac{\mathbf{J}^{-1} * \mathbf{q}}{Y}=\frac{1}{Y}\left(\frac{q_{2}}{I_{x}} \mathbf{e}_{1}-\frac{q_{1}}{I_{y}} \mathbf{e}_{2}\right)
$$


Asymptotic expansions for mono-connected (open) sections

In technical textbooks mono-connected sections are called open, referring to their middle line. It may be proven that the problem (4)-(7) in this case is equivalent to

$$
\begin{aligned}
\Delta \Psi & =0 \quad \text { in } \mathcal{D}^{o}, \\
\operatorname{grad} \Psi \cdot \mathbf{l} & =-\frac{\mathbf{k}}{4} \cdot\left\{\left[\alpha\left(\mathbf{r}_{b} \otimes \mathbf{r}_{b}\right)+\beta\left(* \mathbf{r}_{b} \otimes * \mathbf{r}_{b}\right)\right] \mathbf{l}\right\} \quad \text { on } \partial \mathcal{D}
\end{aligned}
$$

if $\mathbf{t}=G\left\{\left[\beta\left(* \mathbf{r}_{b} \otimes \mathbf{r}_{b}\right)-\alpha\left(\mathbf{r}_{b} \otimes * \mathbf{r}_{b}\right)\right] \frac{\mathbf{k}}{4}-* \operatorname{grad} \Psi\right\}, \Psi$ is a scalar function defined over the section and $\alpha=1-2 \nu, \beta=3+2 \nu$; we use the definition $(\mathbf{x} \otimes \mathbf{y}) \mathbf{z}:=(\mathbf{x} \cdot \mathbf{z}) \mathbf{y}$. Integration of (13) leads to a Dirichlet boundary condition to be added to (12). We then consider (12)-(13) in $\mathcal{D}_{\epsilon}$ with coordinates $s, z$. We denote by $f_{1}, f_{2}$ the restrictions of $\Psi(s, z, \varepsilon)$ to the curves $z=-1, z=1$ respectively, assuming, without loss of generality, $\Psi(0,0)=0$. Laplace operator becomes a linear differential operator whose coefficients are rational functions in $\epsilon([9]) ; f_{1}, f_{2}$ are fifth order polynomials in $\epsilon$. We are so led to assume

$$
\Psi(s, z, \epsilon)=\sum_{n=0}^{\infty} \Psi_{n}(s, z) \epsilon^{n} .
$$

As a consequence, (12)-(13) become

$$
\begin{gathered}
\sum_{n=0}^{\infty}\left\{\epsilon^{n+3} \delta^{3}\left[-z^{3} \kappa^{3} \Psi_{n, z z}-z \kappa \Psi_{n, s s}-z^{2} \kappa^{3} \Psi_{n, z}+z \kappa \Psi_{n, s}\right]+\right. \\
\epsilon^{n+2} \delta^{2}\left[3 z^{2} \kappa^{2} \Psi_{n, z z}+\Psi_{n, s s}+2 z \kappa^{2} \Psi_{n, z}\right]+ \\
\epsilon^{n+1} \delta^{1}\left[-3 z \kappa \Psi_{n, z z}-\kappa \Psi_{n, z}\right]+ \\
\left.\epsilon^{n} \quad\left[\Psi_{n, z z}\right]\right\}=0 \quad \text { in }[0, l] \times[-1,1] \\
\sum_{n=0}^{\infty} \Psi_{n}(s,-1) \epsilon^{n}=f_{1}(\epsilon, s) \quad z=-1, \\
\sum_{n=0}^{\infty} \Psi_{n}(s, 1) \epsilon^{n}=f_{2}(\epsilon, s) \quad z=1
\end{gathered}
$$

$\kappa$ is the curvature of $\mathcal{L}$ and the comma means differentiation with respect to the indicated variables. Equating the first four powers in $\epsilon$ in (15)-(17), we obtain

$$
\begin{aligned}
\Psi_{0}(s, z)= & -\frac{\mathbf{k}}{4} \cdot \int_{0}^{s}\left[\alpha\left(\mathbf{r}_{0} \otimes \mathbf{r}_{0}\right)+\beta\left(* \mathbf{r}_{0} \otimes * \mathbf{r}_{0}\right)\right] \dot{\mathbf{r}}_{0} d s \\
\Psi_{1}(s, z)= & z \delta \frac{\mathbf{k}}{4} \cdot \int_{0}^{s}\left\{\alpha\left[\kappa\left(\mathbf{r}_{0} \otimes \mathbf{r}_{0}\right) \dot{\mathbf{r}}_{0}-*\left(\dot{\mathbf{r}}_{0} \otimes \dot{\mathbf{r}}_{0}\right) \mathbf{r}_{0}\right]+\beta\left[\kappa\left(* \mathbf{r}_{0} \otimes * \mathbf{r}_{0}\right) \dot{\mathbf{r}}_{0}+\left(\mathbf{I}+\dot{\mathbf{r}}_{0} \otimes \dot{\mathbf{r}}_{0}\right) * \mathbf{r}_{0}\right]\right\} d s+ \\
& -z \delta \frac{\mathbf{k}}{4} \cdot\left|\left[\alpha\left(\mathbf{r}_{0} \otimes \mathbf{r}_{0}\right) * \dot{\mathbf{r}}_{0}+\beta *\left(\mathbf{r}_{0} \otimes \mathbf{r}_{0}\right) \dot{\mathbf{r}}_{0}\right]\right|_{s=0} \\
\Psi_{2}(s, z)= & \delta^{2} \frac{\mathbf{k}}{4} \cdot\left\{\left(z^{2}-1\right)\left[4(1+\nu) \kappa \int_{0}^{s} * \mathbf{r}_{0} d s+\left[\alpha\left(\dot{\mathbf{r}}_{0} \otimes \dot{\mathbf{r}}_{0}\right)-(1+2 \nu)\left(* \dot{\mathbf{r}}_{0} \otimes * \dot{\mathbf{r}}_{0}\right)\right] \mathbf{r}_{0}\right]+\right. \\
& -\frac{1}{2}\left|\left[\alpha\left(\mathbf{I}+* \dot{\mathbf{r}}_{0} \otimes * \dot{\mathbf{r}}_{0}\right)-\beta\left(\dot{\mathbf{r}}_{0} \otimes \dot{\mathbf{r}}_{0}\right)\right] \mathbf{r}_{0}\right|_{s=0}+ \\
& +\int_{0}^{s}\left\{\alpha\left[\kappa *\left(\dot{\mathbf{r}}_{0} \otimes \dot{\mathbf{r}}_{0}\right) \mathbf{r}_{0}-\left(\frac{\mathbf{v} \otimes \mathbf{r}_{0}+\mathbf{r}_{0} \otimes \mathbf{v}}{3 l}\right) \dot{\mathbf{r}}_{0}\right]+\right. \\
& \left.\left.-\beta\left[\kappa\left(\mathbf{I}+\dot{\mathbf{r}}_{0} \otimes \dot{\mathbf{r}}_{0}\right) * \mathbf{r}_{0}+\dot{\mathbf{r}}_{0}+\left(\frac{* \mathbf{v} \otimes * \mathbf{r}_{0}+* \mathbf{r}_{0} \otimes * \mathbf{v}}{3 l}\right) \dot{\mathbf{r}}_{0}\right]\right\} d s\right\}
\end{aligned}
$$




$$
\begin{aligned}
\Psi_{3}(s, z)= & \frac{\left(z^{3}-z\right)}{3} \delta^{3} \frac{\mathbf{k}}{4} \cdot\left\{-4 \nu \kappa \mathbf{r}_{0}+8(1+\nu) \kappa^{2} * \int_{0}^{s} \mathbf{r}_{0} d s-(1+2 \nu) * \dot{\mathbf{r}}_{0}\right\}+ \\
& -z \frac{\mathbf{k}}{12} \cdot\left|\alpha\left(\mathbf{I}+\frac{\mathbf{v} \otimes \mathbf{r}_{0}+\mathbf{r}_{0} \otimes \mathbf{v}}{l}\right) * \dot{\mathbf{r}}_{0}+\frac{\beta}{l} *\left(\frac{\mathbf{v} \otimes \mathbf{r}_{0}+\mathbf{r}_{0} \otimes \mathbf{v}}{3 l}\right) \dot{\mathbf{r}}_{0}\right|_{s=0}+ \\
& -z \frac{\mathbf{k}}{4} \int_{0}^{s}\left\{\frac{\alpha}{3 l}\left[*\left(\dot{\mathbf{r}}_{0} \otimes \dot{\mathbf{r}}_{0}\right) \mathbf{v}-\kappa\left(\mathbf{v} \otimes \mathbf{r}_{0}+\mathbf{r}_{0} \otimes \mathbf{v}\right) \dot{\mathbf{r}}_{0}\right]+\right. \\
& \left.-\frac{\beta}{3 l}\left[\left(\mathbf{I}+\dot{\mathbf{r}}_{0} \otimes \dot{\mathbf{r}}_{0}\right) * \mathbf{v}+\kappa\left(* \mathbf{v} \otimes * \mathbf{r}_{0}+* \mathbf{r}_{0} \otimes * \mathbf{v}\right) \dot{\mathbf{r}}_{0}+3 l \kappa \dot{\mathbf{r}}_{0}\right]\right\} d s
\end{aligned}
$$

Because of the relationship between $\mathbf{t}$ and $\Psi$ and taking into account the expression of the gradient operator in $s, z$ coordinates ([9]), we obtain

$$
\begin{aligned}
\mathrm{t}_{0}= & Y\left(\mathbf{k} \cdot * \int_{0}^{s} \mathbf{r}_{0} d s\right) \dot{\mathbf{r}}_{0}=-\frac{1}{2 \delta}\left(\frac{q_{1} S_{y}}{I_{y}}+\frac{q_{2} S_{x}}{I_{x}}\right) \dot{\mathbf{r}}_{0}=: t_{s 0} \dot{\mathbf{r}}_{0} \\
\mathbf{t}_{1}= & 2 z G \delta\left\{\mathbf{k} \cdot\left[(1+\nu) \kappa * \int_{0}^{s} \mathbf{r}_{0} d s-\nu \mathbf{r}_{0}\right]\right\} \dot{\mathbf{r}}_{0}=z \delta\left[\kappa t_{s 0}-\frac{\nu}{1+\nu}\left(\frac{q_{2} x(s)}{I_{x}}-\frac{q_{1} y(s)}{I_{y}}\right)\right] \dot{\mathbf{r}}_{0}, \\
\mathbf{t}_{2}= & G \delta^{2}\left\{\left(1-z^{2}\right)\left[(1+\nu)\left(\kappa, s * \int_{0}^{s} \mathbf{r}_{0} d s+2 \kappa * \mathbf{r}_{0}\right)+\dot{\mathbf{r}}_{0}\right] \otimes * \dot{\mathbf{r}}_{0}+\right. \\
& \left.+\left[\frac{3 z^{2}-1}{3}\left(2(1+\nu) \kappa^{2} * \int_{0}^{s} \mathbf{r}_{0} d s-\nu\left(\kappa \mathbf{r}_{0}+* \dot{\mathbf{r}}_{0}\right)\right)+\frac{2(1+\nu)}{3} \int_{0}^{s}\left(\kappa \dot{\mathbf{r}}_{0}+* \frac{\mathbf{v}}{l}\right) d s\right] \otimes \dot{\mathbf{r}}_{0}\right\} \mathbf{k}= \\
= & \delta^{2}\left(z^{2}-\frac{1}{3}\right)\left\{\kappa^{2} t_{s 0}-\frac{\nu}{2(1+\nu)}\left[\kappa\left(\frac{q_{1} y(s)}{I_{y}}-\frac{q_{2} x(s)}{I_{x}}\right)+\left(\frac{q_{1}}{I_{y}} \mathbf{e}_{1}-\frac{q_{2}}{I_{x}} \mathbf{e}_{2}\right)\right] \cdot \dot{\mathbf{r}}_{0}\right\} \dot{\mathbf{r}}_{0}+ \\
& +\frac{2}{3} \delta^{2}\left\{\left(\frac{q_{2}}{I_{x}} \mathbf{e}_{2}-\frac{q_{1}}{I_{y}} \mathbf{e}_{1}\right) \cdot \int_{0}^{s}\left(\kappa \dot{\mathbf{r}}_{0}+* \frac{\mathbf{v}}{l}\right) d s\right\} \dot{\mathbf{r}}_{0}+ \\
& +\left(1-z^{2}\right) \delta^{2}\left\{\frac{\kappa, s}{2} t_{s 0}-\kappa\left(\frac{q_{1} x(s)}{I_{y}}+\frac{q_{2} y(s)}{I_{x}}\right)+\frac{1}{2(1+\nu)}\left(e_{1} \frac{q_{2}}{I_{x}}-\frac{q_{1}}{I_{y}} \mathbf{e}_{2}\right) \cdot \dot{\mathbf{r}}_{0}\right\} * \dot{\mathbf{r}}_{0} .
\end{aligned}
$$

In the former, $S_{x}, S_{y}$ are the first moment of area of the portion of the section corresponding to a cut directed along $* \dot{\mathrm{r}}_{0}$ at the arc length $s$, with respect to the $x$ and $y$ axes; $x(s), y(s)$ are the parametric equations of the middle line. Equation (22) is Jourawski formula; note that $\int_{0}^{l} \mathbf{r}_{0} d s=\mathbf{0}$ because of the choice of the centroid as origin of $\mathcal{P}$. We now compare the formal $\epsilon$-series expansions of the right hand sides of equalities $(9)_{1}$ and $(9)_{2}$, whose coefficients we denote $c_{n}, d_{n}$ respectively. As obtained by equality $(9)_{2}$, the resultant shear stress is a sixth order polynomial in $\epsilon$ whose even coefficients vanish. On the contrary, if we replace in (9) $)_{1}$ the formal expansion for $\boldsymbol{t}$, in general we obtain a complete power series in $\epsilon$. This is what we call effect of Kelvin type in flexure (see [11] for Kelvin effect in torsion). We estimate this effect by means of the difference $\mathbf{c}_{n}-\mathbf{d}_{n}$ for $n=1,2,3$. We have, denoting by $\mathbf{J}_{b n}$ the $n$-th term of $\mathbf{J}_{b}$ induced by (3),

$$
\begin{aligned}
& \mathbf{c}_{1}=\mathbf{d}_{1}=Y *\left[2 \delta \int_{0}^{l}\left(* \mathbf{r}_{0} \otimes * \mathbf{r}_{0}\right) d s\right] \mathbf{k}=Y * \mathbf{J}_{b 1} \mathbf{k}, \\
& \mathbf{c}_{2}=\mathbf{d}_{2}=\mathbf{0} \\
& \mathbf{c}_{3}-\mathbf{d}_{3}=-\frac{4}{3} G \delta^{3} \nu\left|\mathbf{r}_{0} \otimes * \dot{\mathbf{r}}_{0}\right|_{0}^{l} \mathbf{k} .
\end{aligned}
$$

The effect appears at first with $\mathrm{c}_{3}$, in relation with $\mathrm{t}_{2}$ (order $\epsilon^{2}$ ): a boundary layer (named edge effect in [8]) arises and its global effect is likely to be given by (27) (note that $\left.\int_{0}^{l}\left(\kappa \dot{\mathbf{r}}_{0}+* \frac{\mathbf{v}}{l}\right) d s=\mathbf{0}\right)$ ). Indeed: a) it may be proven that the $\Psi_{n}$ we have given verify exactly all boundary conditions up to order one in $\epsilon$; b) the field $t_{0}$ verifies boundary condition (6) pointwise, $t_{1}$ and $t_{2}$ as a mean over the thickness. 
Asymptotic expansions for bi-connected (closed) sections

The Helmholtz problem (4)-(7) can be turned into Poisson form with Dirichlet boundary conditions once a particular solution of (4) and (7) is found. Such a solution is not uniquely determined for bi-connected sections; in order to avoid a gauge choice whose physical meaning is uncertain, we apply a perturbation method directly to (4)-(7). In $s, z$ coordinates ([9]), equations (4)-(7) become (here $0 \approx l$ )

$$
\begin{aligned}
\epsilon \delta t_{s, s}+(1-z \kappa \epsilon \delta) t_{z, z}-\epsilon \delta \kappa t_{z} & =\epsilon \delta(1-z \kappa \epsilon \delta) Y \mathbf{k} \cdot\left(* \mathbf{r}_{0}-z \epsilon \delta \dot{\mathbf{r}}_{0}\right) \quad \text { in }[0, l] \times[-1,1], \\
\epsilon \delta t_{z, s}-(1-z \kappa \epsilon \delta) t_{s, z}+\epsilon \delta \kappa t_{s} & =2 \epsilon \delta(1-z \kappa \epsilon \delta) G \nu \mathbf{k} \cdot\left(\mathbf{r}_{0}+z \epsilon \delta * \dot{\mathbf{r}}_{0}\right) \quad \text { in }[0, l] \times[-1,1] \\
t_{z} & =0 \quad z= \pm 1 \\
\int_{\mathcal{L}} t_{s} d s & =2 G \nu \mathbf{k} \cdot A_{\mathcal{R}}\left(b_{\mathcal{R}}-m\right) \quad z=0
\end{aligned}
$$

in the former $\mathbf{t}=t_{s} \dot{\mathbf{r}}_{0}+t_{z} * \dot{\mathbf{r}}_{0}$; because of $(8), b=m$. We propose the formal expansion

$$
\mathrm{t}=\dot{\mathbf{r}}_{0} \sum_{n=0}^{\infty} t_{s n} \epsilon^{n}+* \dot{\mathbf{r}}_{0} \sum_{n=0}^{\infty} t_{z n} \epsilon^{n}
$$

Substituting (32) into (28)-(31) we obtain a hierarchy of problems for $\mathbf{t}$. Equating first powers in $\epsilon$ we have:

$$
\begin{aligned}
\mathbf{t}_{0}= & \left\{Y \mathbf{k} \cdot\left[\int_{0}^{s} * \mathbf{r}_{0} d s-\frac{1}{l} \int_{\mathcal{L}}\left(\int_{0}^{s} \mathbf{r}_{0} d s\right) d s\right]+\frac{2 G A_{\mathcal{R}} \nu \mathbf{k} \cdot\left(b_{\mathcal{R}}-m\right)}{l}\right\} \dot{\mathbf{r}}_{0}= \\
= & \left\{-\frac{1}{2 \delta}\left(\frac{q_{1} S_{y}}{I_{y}}+\frac{q_{2} S_{x}}{I_{x}}\right)+\frac{\nu}{l(1+\nu)}\left(\frac{q_{2} S_{\mathcal{R}_{x}}}{I_{x}}-\frac{q_{1} S_{\mathcal{R}_{y}}}{I_{y}}\right)\right\} \dot{\mathbf{r}}_{0}=: t_{s 0} \dot{\mathbf{r}}_{0}, \\
\mathbf{t}_{1}= & z \delta\left[\kappa t_{s 0}-2 G\left(\nu \mathbf{k} \cdot \mathbf{r}_{0 m}\right)\right] \dot{\mathbf{r}}_{0}=z \delta\left\{\kappa t_{s 0}-\frac{\nu}{1+\nu}\left[\frac{q_{2} x_{m}(s)}{I_{x}}-\frac{q_{1} y_{m}(s)}{I_{y}}\right]\right\} \dot{\mathbf{r}}_{0}, \\
\mathrm{t}_{2}= & \delta^{2}\left\{\left(1-z^{2}\right)\left[\frac{\kappa, s}{t_{s 0}}+\mathbf{k} \cdot\left(G \dot{\mathbf{r}}_{0}+Y \kappa * \mathbf{r}_{0 m}\right)\right] * \dot{\mathbf{r}}_{0}+\right. \\
& \left.+\left[\left(\frac{3 z^{2}-1}{3}\right)\left(\kappa^{2} t_{s 0}+\nu G \mathbf{k} \cdot\left(\kappa \mathbf{r}_{0}+* \dot{\mathbf{r}}_{0}\right)\right)-\frac{Y}{3} \mathbf{k} \cdot * \dot{\mathbf{r}}_{0}+\frac{1}{3 l} \int_{\mathcal{L}} \kappa\left[\kappa t_{s 0}-G\left(\nu \mathbf{k} \cdot \mathbf{r}_{0}\right)\right] d s\right] \dot{\mathbf{r}}_{0}\right\}= \\
= & \left(1-z^{2}\right) \delta^{2}\left\{\frac{\kappa, s}{2} t_{s 0}+\frac{1}{2(1+\nu)}\left(\frac{q_{2}}{I_{x}} \mathbf{e}_{1}-\frac{q_{1}}{I_{y}} \mathbf{e}_{2}\right) \cdot \dot{\mathbf{r}}_{0}-\kappa\left[\frac{q_{2} y(s)}{I_{x}}+\frac{q_{1} x(s)}{I_{y}}\right]\right\} * \dot{\mathbf{r}}_{0}+ \\
& +\delta^{2}\left\{\left(\frac{3 z^{2}-1}{3}\right)\left[\kappa^{2} t_{s 0}+\frac{\nu}{2(1+\nu)}\left[\kappa\left(\frac{q_{1} y(s)}{I_{y}}-\frac{q_{2} x(s)}{I_{x}}\right)+\left(\frac{q_{1}}{I_{y}} \mathbf{e}_{1}+\frac{q_{2}}{I_{x}} \mathbf{e}_{2}\right) \cdot \dot{\mathbf{r}}_{0}\right]\right]+\right. \\
& \left.+\frac{1}{3}\left(\frac{q_{1}}{I_{y}} \mathbf{e}_{1}+\frac{q_{2}}{I_{x}} \mathbf{e}_{2}\right) \cdot \dot{\mathbf{r}}_{0}+\frac{1}{3 l} \int\left[\kappa_{\mathcal{C}} t_{s 0}-\frac{\nu \kappa}{2(1+\nu)}\left(\frac{q_{1} y(s)}{I_{y}}-\frac{q_{2} x(s)}{I_{x}}\right)\right] d s\right\} \dot{\mathbf{r}}_{0} .
\end{aligned}
$$

In the former, $S_{\mathcal{R} x}, S_{\mathcal{R}_{y}}$ are the first moment of area of the region $\mathcal{R}$ with respect to the $x$ and $y$ axes; $x(s), y(s)$ are the parametric equations of the middle line with respect to the point $m$. Equation (33) is Jourawski formula for closed sections; note that the second term in the right hand side of (33) gives a Bredt shear stress field for closed sections of constant thickness, once the product $\nu \mathbf{k} \cdot\left(b_{\mathcal{R}}-m\right)$ is interpreted as a unit angle of twist. In technical literature (33) is often given in implicit form, as a consequence of the principle of virtual work.

Once introduced $c_{n}, d_{n}$ with the same meaning as in the previous section we have

$$
\begin{aligned}
& \mathbf{c}_{1}=\mathbf{d}_{1}=Y *\left[2 \delta \int_{0}^{l}\left(* \mathbf{r}_{0} \otimes * \mathbf{r}_{0}\right) d s\right] \mathbf{k}=Y * \mathbf{J}_{b 1} \mathbf{k} \\
& \mathbf{c}_{2}=\mathbf{d}_{2}=\mathbf{0} \\
& \mathbf{c}_{3}=\mathbf{d}_{3}=Y *\left\{\frac{2}{3} \delta^{3} \int_{0}^{l}\left[\kappa\left(\dot{\mathbf{r}}_{0} \otimes * \mathbf{r}_{0}+* \mathbf{r}_{0} \otimes \dot{\mathbf{r}}_{0}\right)+\left(\dot{\mathbf{r}}_{0} \otimes \dot{\mathbf{r}}_{0}\right)\right] d s\right\} \mathbf{k}=Y * \mathbf{J}_{b 3} \mathbf{k}
\end{aligned}
$$


In closed sections the expansions $(9)_{1},(9)_{2}$ lead to the same results at the first three orders in $\epsilon$.

\section{Applications and conclusions}

We start considering three sections of particular technological interest (see for instance [6], [7], [12], [13]), rectangular and semicircular (open) and annular (closed).

Rectangle

We have $b=m$ and zero curvature of the middle line; given an orthonormal basis $\mathbf{e}_{1}, \mathrm{e}_{2}$ for $\mathcal{P}$, it is

$$
\mathbf{r}=(s-h) \mathbf{e}_{1}+z \epsilon \delta \mathbf{e}_{2} \Rightarrow \dot{\mathbf{r}}_{0}=\mathrm{e}_{1}, * \dot{\mathbf{r}}_{0}=\mathbf{e}_{2},
$$

where $h$ is half the length of the middle line. Substituting (39) into (22)-(24) we have

$$
\begin{aligned}
& \mathbf{t}_{0}=\frac{q_{1}}{I_{y}}\left(h s-\frac{s^{2}}{2}\right) \mathbf{e}_{1}, \\
& \mathbf{t}_{1}=\frac{z \delta(s-h)}{1+\nu} \frac{q_{2}}{I_{x}} \mathbf{e}_{1}, \\
& \mathbf{t}_{2}=\frac{\delta^{2}}{2(1+\nu)}\left[\nu\left(\frac{3-z^{2}}{3}\right) \frac{q_{1}}{I_{y}} \mathbf{e}_{1}+\left(1-z^{2}\right) s \frac{q_{2}}{I_{x}} \mathbf{e}_{2}\right] .
\end{aligned}
$$

The resultant shear forces and the estimate of Kelvin effect are given by

$$
\begin{aligned}
& \mathrm{c}_{1}=\mathrm{d}_{1}=Y^{\prime} \frac{4}{3} \delta h^{3}\left(\mathbf{e}_{2} \otimes \mathbf{e}_{1}\right) \mathbf{k}=Y * \mathbf{J}_{b 1} \mathbf{k}, \\
& \mathbf{c}_{2}=\mathrm{d}_{2}=0 \\
& \mathbf{c}_{3}-\mathrm{d}_{3}=\frac{8}{3} G \delta^{3} h\left(\mathbf{e}_{1} \otimes \mathbf{e}_{2}\right) \nu \mathbf{k}=\frac{\nu}{1+\nu} q_{2} \mathbf{e}_{2} .
\end{aligned}
$$

Eq. (40) gives the Jourawski field ([6], [7], [11], [12], [13]). Eq. (41) implies that when $\mathbf{k} / / \mathbf{e}_{2}\left(\mathbf{q} / / \mathbf{e}_{1}\right)$ the first order correction to Jourawski formula vanishes, which is a known result. On the other hand, if $\mathbf{k} / / \mathbf{e}_{1}$ eq. (41) gives a nonvanishing correction to Jourawski formula, bilinear in $(s-h)$ and $z$, result which we could not find in literature. Eq. (42) supplies the estimate of the second order correction to eq. (40) given by our perturbation method and gives an analytical expression for the shear stress field arising in flexure of 'thick' rectangles. It may be easily proven that, if the material of which the section is made has $\nu=1 / 3$ (as it is, in average, for steel, for example), one has:

a. if the stress distribution is equivalent to a force parallel to $e_{1}$,

$$
\frac{t_{2, \max }}{t_{0, \max }}=\frac{\delta^{2}}{6 h^{2}}= \begin{cases}0.17 \%, & \text { if } \delta / h=1 / 10 \\ 0.7 \%, & \text { if } \delta / h=1 / 5 \\ 1.85 \%, & \text { if } \delta / h=1 / 3 \\ 4.27 \%, & \text { if } \delta / h=1 / 2\end{cases}
$$

b. if the stress distribution is equivalent to a force parallel to $\mathbf{e}_{2}$,

$$
\frac{t_{2, \max }}{t_{1, \max }}=\frac{\delta}{2 h}= \begin{cases}5 \%, & \text { if } \delta / h=1 / 10 \\ 10 \%, & \text { if } \delta / h=1 / 5 \\ 16 \%, & \text { if } \delta / h=1 / 3 \\ 25 \%, & \text { if } \delta / h=1 / 2\end{cases}
$$

The difference appearing in eq. (45) vanishes when $\mathbf{k} / / \mathbf{e}_{2}\left(\mathbf{q} / / \mathbf{e}_{1}\right)$ : Kelvin effect in flexure arises only when $\mathbf{k} / / \mathbf{e}_{1}\left(\mathbf{q} / / \mathbf{e}_{2}\right)$. We remark that

$$
\mathbf{c}_{3}-\mathbf{d}_{3}=\frac{G(2 \delta)^{3}}{3} \tau_{1} \mathbf{e}_{2}, \quad \tau_{1}:=\nu \mathbf{k} \cdot\left(h \mathbf{e}_{1}\right) ;
$$


$2 \delta$ is the section thickness. Kelvin effect in torsion is given by (46) when $\tau_{1}$ is the unit angle of twist.

\section{Semicircular section}

Given an orthonormal basis $\mathbf{e}_{1}, \mathbf{e}_{2}$ for $\mathcal{P}$, eq. (1), with respect to the centre of the circle, is

$$
\mathbf{r}=(r-z \epsilon \delta)\left(\cos \frac{s}{r} \mathbf{e}_{1}+\sin \frac{s}{r} \mathbf{e}_{2}\right), \quad s \in[0, l=\pi r], z \in[-1,1],
$$

where $r$ is the radius of the middle line. Its centre of position is given by the vector $m-o=\frac{2 r}{\pi} \mathbf{e}_{2}$. As there are no qualitative difference among the fields $t_{n}$ for this case and for the rectangle, we give only the estimate of the Kelvin effect at order three in $\epsilon$ :

$$
\mathbf{c}_{3}-\mathbf{d}_{3}=\frac{16}{3 \pi} G \delta^{3} r\left(\mathbf{e}_{2} \otimes \mathbf{e}_{1}\right) \nu \mathbf{k} .
$$

In this case, the difference in (48) vanishes when $k / / e_{1}\left(q / / e_{2}\right)$ and Kelvin effect in flexure arises only when $\mathbf{k} / / \mathbf{e}_{2}\left(\mathbf{q} / / \mathbf{e}_{1}\right)$. Again, we remark that

$$
\mathrm{c}_{3}-\mathbf{d}_{3}=\frac{G(2 \delta)^{3}}{3} \tau_{2} \mathbf{e}_{2}, \quad \tau_{2}:=\nu \mathbf{k} \cdot\left(\frac{2 r}{\pi} \mathbf{e}_{1}\right)
$$

$2 \delta$ is the section thickness. Kelvin effect in torsion is given by (49) when $\tau_{2}$ is the unit angle of twist.

\section{Circular tube}

We have $b=m$ and constant curvature of $\mathcal{L}_{\text {; }}$ with respect to the orthonormal basis $\mathbf{e}_{1}, \mathbf{e}_{2}$, eq. (1) is

$$
\mathrm{r}=(r-z \epsilon \delta)\left(\cos \frac{s}{r} \mathbf{e}_{1}+\sin \frac{s}{r} \mathbf{e}_{2}\right), \quad s \in[0, l=2 \pi r], z \in[-1,1]
$$

$r$ is the radius of the middle circumference, so that $\kappa=1 / r$. We have the following:

$$
\begin{aligned}
& \mathbf{t}_{0}=r^{2}\left(\frac{q_{2}}{I_{x}} \cos \frac{s}{r}-\frac{q_{1}}{I_{y}} \sin \frac{s}{r}\right) \dot{\mathbf{r}}_{0}, \\
& \mathbf{t}_{1}=\frac{z \delta r}{1+\nu}\left(\frac{q_{2}}{I_{x}} \cos \frac{s}{r}-\frac{q_{1}}{I_{y}} \sin \frac{s}{r}\right) \dot{\mathbf{r}}_{0}, \\
& \mathbf{t}_{2}=G \delta^{2}\left[\left(1-z^{2}\right) \beta\left(\frac{q_{2}}{Y I_{x}} \sin \frac{s}{r}-\frac{q_{1}}{Y I_{y}} \cos \frac{s}{r}\right) * \dot{\mathbf{r}}_{0}-\frac{z^{2}}{2 G} \dot{\mathbf{r}}_{0}\right]\left(\frac{q_{2}}{I_{x}} \cos \frac{s}{r}-\frac{q_{1}}{I_{y}} \sin \frac{s}{r}\right) .
\end{aligned}
$$

These fields are the same as these given by a truncated expansion of the exact analytic solution ([6]). Our series cannot supply the exact solution with a finite number of iteration steps because the exact solution is rational in $\epsilon$; on the other hand, we had no need of introducing complex variables and harmonic functions. Eqs. (51)-(53), once integrated along the thickness, give the fields plotted in [12]. As previously made for the rectangle, assuming $\nu=1 / 3$ one has:

a. comparing the stress component along $\dot{\mathbf{r}}_{0}$,

$$
\frac{t_{1, \max }}{t_{0, \max }}=\frac{\delta}{r(1+\nu)}= \begin{cases}7.5 \%, & \text { if } \delta / r=1 / 10 \\ 15 \%, & \text { if } \delta / r=1 / 5 \\ 25 \%, & \text { if } \delta / r=1 / 3 \\ 37.5 \%, & \text { if } \delta / r=1 / 2\end{cases}
$$

b. comparing the stress component along $* \dot{\mathbf{r}}_{0}$.

$$
\frac{t_{2, \max }}{t_{1, \max }}=\frac{\delta^{2}}{2 r^{2}}= \begin{cases}0.5 \%, & \text { if } \delta / r=1 / 10 \\ 2.0 \%, & \text { if } \delta / r=1 / 5 \\ 5.5 \%, & \text { if } \delta / r=1 / 3 \\ 12.5 \%, & \text { if } \delta / h=1 / 2\end{cases}
$$

The $c_{n}, d_{n}$ are

$$
\begin{aligned}
& \mathbf{c}_{1}=\mathbf{d}_{1}=Y *\left(2 \pi r^{3} \delta\right)\left(\mathbf{e}_{1} \otimes \mathbf{e}_{1}+\mathbf{e}_{2} \otimes \mathbf{e}_{2}\right) \mathbf{k}=Y * \mathbf{J}_{1} \mathbf{k} \\
& \mathbf{c}_{2}=\mathbf{d}_{2}=\mathbf{0} \\
& \mathbf{c}_{3}=\mathbf{d}_{3}=Y *\left(2 \pi r \delta^{3}\right)\left(\mathbf{e}_{1} \otimes \mathbf{e}_{2}-\mathbf{e}_{2} \otimes \mathbf{e}_{1}\right) \mathbf{k}=Y * \mathbf{J}_{3} \mathbf{k}
\end{aligned}
$$


In this case, the shear stress resultants as obtained by $(9)_{2}$ and $(9)_{3}$ are exactly the same, which is a result to be expected because of the particularly rich symmetry group of the considered section.

\section{Comparison with Vlasov theory of thin walled bars}

We have proposed a perturbation method that has given us the possibility of finding simple formulæ that provide higher order corrections to the known Jourawski shear stress field, both for mono- and bi-connected sections of a Saint-Venant cylinder with constant thickness.

We remark that:

a) we have introduced no constraints of Kirchhoff type to obtain our formulæ, while in [12] such constraint is the basis for all the presented results; on the contrary, we have proven that at the first order in $\epsilon$ Kirchhoff constraint is automatically satisfied. This fact has a natural and immediate interpretation in terms of our procedure, because the lowest step of the asymptotic expansion stands for a thin section, in the ordinary sense attributed to this term in the technical literature. In this case, the result naturally found by us which needs to be postulated by means of an internal constraint is physically reasonable and meaningful;

b) the proposed perturbative method allows for a precise analysis of the relative order of magnitude of the shear stress field in thin walled bars in terms of a thickness parameter of the section; on the contrary, the analysis developed in [12] leads to the determination of only the first nonzero fields by means of a procedure involving reactive forces and shell-like models. We have also shown, in some simple but significant cases from the point of view of technological applications, that our simple formulæ provide nonzero corrections to the Jourawski shear stress field that may be quite relevant when the section becomes thick. In the literature, only in few cases -e.g. annular and rectangular sections- higher order corrections can be evaluated considering series expansions, while the expansion method presented here can be applied for every Bredt-like section.

\section{References}

[1]. L. Wheeler, Stress bounds for the torsion of tubes of uniform wall thickness, J. of Elasticity 4 (1974), 281-292

[2]. L. Wheeler, C. O. Horgan, Upper and lower bounds for the shear stress in the Saint-Venant theory of flexure, J. of Elasticity 6 (1976), 383-403

[3]. F. dell'Isola, G. C. Ruta, Outlooks in Saint-Venant theory I: formal expansions for torsion of Bredt-like sections, Arch. Mech. 46 (1994), 1005-1027

[4]. F. dell'Isola, L. Rosa, Perturbation methods in torsion of thin hollow Saint-Venant cylinders, Mech. Res. Comm. 23 (1996), 145-150

[5]. A. Clebsch, Théorie de l'élasticité des corps solides (Traduite par MM. Barré de Saint-Venant et Flamant, avec des notes étendues de M. Barré de Saint-Venant), Dunod, Paris (1883), reprinted by Johnson Reprint Corporation, New York (1966)

[6]. B. M. Fraeijs de Veubeke, A course in elasticity, Springer-Verlag, New York (1979)

[7]. G. Ceradini, Teoria della trave, ESA, Roma (1989)

[8]. A. Nayfeh, Perturbation methods, John Wiley and Sons, New York (1973)

[9]. V. I. Arnold, Metodi matematici della meccanica classica, Editori Riuniti, Roma (1992)

[10]. K. Frischmuth, M. Haenler, F. dell'Isola, Numerical Methods versus asymptotic expansion for torsion of hollow elastic beams, Fachbereich Mathematik der Universität Rostock 20/95 (1995)

[11]. S. Timoshenko, J. N. Goodier, Theory of elasticity, McGraw-Hill, New York (1951)

[12]. A. Gjelsvik, The theory of thin walled bars, John Wiley and Sons, New York (1981)

[13]. V. I. Feodosev, Strength of Materials, MIR publishers, Moscow (1968) 